MEDICAL EDUCATION

\title{
The research component of specialist registration - a question of alligators and swamps? A personal view
}

\begin{abstract}
C M Aldous, M Adhikari, C C Rout
Colleen Aldous is a senior lecturer in the School of Clinical Medicine at the University of KwaZulu-Natal, Durban, South Africa. She is a medical scientist with a PhD in science education and is involved in postgraduate research mentorship across several medical disciplines including surgery, orthopaedics, dermatology, paediatrics, opthalmology, general medicine and psychology. Her own research interest is human genetics, and she is a member of the national steering committee and working group reviewing National Department of Health policy guidelines for human genetics services. Miriam Adhikari, MB ChB, FCP (Paeds), PhD, is currently Scientific Advisor to the Postgraduate Office, School of Clinical Medicine at UKZN. She has had a long and distinguished research career at the UKZN medical school and has received several awards and accolades for her long-term and continued service to paediatrics in the province. She has served on several Health Professions Council of South Africa committees across the country, and has a wealth of experience in postgraduate education. Chris Rout, MB BS, FFARCS, FCA (SA), is in private anaesthetic practice in Durban. He maintains a research interest in obstetric anaesthesia and is developing interests in clinical skills transfer, clinical support needs of medical officers in rural district hospitals, and the influence of Plato on 15th century Florentine art.
\end{abstract}

Corresponding author: C M Aldous (colleenaldous@gmail.com)

The recent implementation of the research requirement for specialist registration presents difficulties with regard to the provision of research supervision, particularly in those medical schools that previously followed the path of qualification via the Colleges of Medicine of South Africa examinations. The differences between the requirements for research supervision as stated in the Health Professions Council of South Africa memorandum and those of the Committee for Higher Education are causing disparities between medical schools similar to those that led to the memorandum in the first place. While the research component of specialist training can only improve the quality of both patient care and academic endeavour, it requires an enormous investment of time on the part of both the specialist trainees and their supervisors. In order to deal with this, specific issues outlined in the article need to be addressed.

S Afr Med J 2015;105(1):21-22. DOI:10.7196/SAMJ.8732

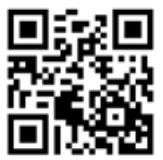

In January 2010, the Medical Subcommittee for Postgraduate Education and Training of the Health Professions Council of South Africa (HPCSA) published new requirements for specialist registration in South Africa (SA) ${ }^{[1]}$ This was to solve the problem of variations between training centres in integrated assessment, variations between exit outcomes 1 and 2 (associated with differences between several MMed centres and the national Colleges of Medicine), and failure to comply with outcome 3 to undertake and complete a relevant research study.

The solution was a common exit examination (currently the Fellowship of the Colleges of Medicine), strict application of the portfolio requirement, and completion of a research component before specialist registration could occur. The research would be completed 'under the supervision of the Head of Department or nominee' and assessed to show that 'appropriate theoretical knowledge is demonstrated; a research protocol is compiled according to required norms; a progress report on the research project is given on a regular basis; and that research results are reported in a format of a dissertation according to acceptable scientific norms. The research study would be allocated a minimum of 60 credits in terms of the Standards Generating Body of the Medical and Dental Board of the HPCSA, would be 'assessed at university level', and 'may be used as a credit for Part III of the MMed degree.[1]

This excellent solution to the pre-existing variations in qualifications for specialist registration has, however, introduced difficulties for some training centres. These include finding supervisors who fulfil regulatory requirements, provision of adequate research time for specialist trainees, and provision of time for supervision.

Although under the control of the HPCSA, post numbers allocated to specialist training are administered by training institutions (effectively university departments). Irrespective of the HPCSA ruling, universities must abide by Committee for Higher Education (CHE) postgraduate policies, procedures and regulations. ${ }^{[2]}$ These state that the criteria for selection and appointment of supervisors, in addition to their being acceptable to the research community, must include the following:

- The supervisor has a qualification in a relevant field of study higher than, or at least at the same level as, the exit level of the postgraduate programme he/she is supervising.

- The supervisor has an appropriate research track record, as well as experience, expertise and peer recognition in the field of study.

- In the case of inexperienced or new supervisors, there is ongoing staff development and support, and joint supervision is explored as an option.

Although some centres have fared better than others, potential supervisors meeting all qualifying criteria are currently scarce in many medical school departments. The unknown extent of this problem has been highlighted in a recent editorial. ${ }^{[3]}$

This scarcity is due partly to recent challenges to specialist training in $\mathrm{SA}^{[3]}$ and partly to specialists having previously developed their experience and expertise in tandem with research relevant to their specialty, but without acquiring degrees. A wellpublished clinician would be rewarded with seniority, often a professorship, and might be head of a department with only the fellowship diploma as the highest documented level of scholarship. Should these highly accomplished academics now be considered as inadequately qualified to supervise MMed students because they do not have a master's degree? 
It could be argued that the research environment, encompassed by the CHE Criteria for Programme Accreditation, ${ }^{[2]}$ is more important than supervisors' paper qualifications. Master's and doctorate degrees are markers of learning achievement; they are not markers of research or teaching (supervision) excellence. Neither guarantees development as a successful research supervisor or an 'appropriate research track record, experience, expertise and peer recognition, although those with $\mathrm{PhD}$ qualifications might be expected to pursue a research track record through future research in their fields.

It takes time for a student to produce the required quality of research, time for good supervision, and time to create more supervisors.

Brenda Wingfield ${ }^{[4]}$ applied the '10 000 hours hypothesis' ${ }^{[5]}$ to argue that it should take 10000 hours of focused and productive work to complete a $\mathrm{PhD}$ (i.e. become an expert in a highly focused topic), 4000 hours for an MSc degree, and 2000 hours for an honours degree. The South African Qualifications Authority equates one credit with 10 notional hours of learning ${ }^{[6]}$ that are supposed to approximate the time the student requires to achieve learning outcomes, assuming a 45-week full-time academic year. In the case of the research component of both HPCSA and CHE regulations, this amounts to a minimum of 60 credits, equivalent to 600 hours' work. This is at variance with Wingfield's estimate, largely because the regulations relate to minimum requirements and her figures relate to reality.

We argue that both figures are underestimates, given the required learning outcomes (producing a scientifically sound research protocol, conducting research and then presenting the research in a scientifically accepted manner) and the lack of research experience that registrars have when they commence their specialist training. The further burden borne by specialist trainees of acquiring required clinical skills and expertise, in addition to an extensive theoretical background, is acknowledged by the CHE as a 'permitted exception' where professional registration in health sciences '... has a credit load far in excess of the indicative 180 credits that the HEQSF [Higher Education Qualifications Sub-Framework] requires for a Master's degree $^{\text {'[6] }}$ (appendix 1, p. 42).

Wingfield ${ }^{[7]}$ also showed that to supervise a research project takes about $10 \%$ of the time that the student has to commit to complete the degree. Even using the above minimum 600 hours allocated to the student, this equates to 60 hours of supervisor commitment per specialist trainee. Although this might be averaged over 4 years, examination preparation and clinical commitments restrict the time available. Supervision has to be fitted into an already crowded schedule of teaching, personal research and clinical service. Firstclass teaching and first-class research are both full-time jobs ${ }^{[8]}$ that, for a clinician, have to be added to his or her third full-time job of patient care. We argue that the figures are gross underestimates of the time necessary for effective supervision, even with well-motivated students. It should be borne in mind that many specialist trainees have no intrinsic interest in research; rather, its pursuance is viewed as a compulsory component of their training, leading to specialist professional qualification.

In the natural sciences it could take as little as 9 years from admission as an undergraduate to achieve a doctorate, which would represent the start of establishing 'an appropriate research track record, as well as experience, expertise and peer recognition in the field of study'; this hypothetically might take an additional 3 years as a full-time academic. In medicine the equivalent figures would be at least 16 years to achieve a $\mathrm{PhD}$ (assuming release from patient care for part of the time) and, considering continuing commitment to clinical work, at least 5 years to establishing a track record, etc. Clearly any mitigation of the current lack of CHE-compliant supervisors is not going to happen overnight.

The unacknowledged reality is that each medical school is coming to grips with the issue of accommodating both CHE and HPCSA supervisor regulations in different ways; one might insist on the master's/ $\mathrm{PhD}$ rule and ignore requirements for research track record, etc.; another might ignore the CHE regulations and combine Fellowship of the Colleges of Medicine with two first-author publications; yet another might trust individual department heads to pick and choose. In short, we are heading back to the situation of separate processes leading to specialist registration.

If the current inconsistencies between regulatory bodies' rules represent the alligators snapping at us, the reasons behind the recent change in HPCSA regulations represent the swamp we are currently forgetting to drain.

The research component, either in its HPCSA or CHE guise, is an important addition to the specialist training curriculum. It can only improve the quality of both patient care and academic endeavour. However, it requires an enormous investment of time on the part of both the specialist trainees and their supervisors, of whom, with regard to the latter, there is a shortage.

The following issues should be addressed:

- The Department of Health should recognise the academic requirements of specialist training and allocate time for this to both trainees and their supervisors in order to comply with the requirements of the HPCSA.

- The HPCSA and CHE should start reading from the same page regarding research supervision. Descriptors such as 'appropriate research track record', etc. must be defined. The process should allow for awarding both the Fellowship and the MMed to our specialist trainees, which they richly deserve.

- A way should be found to incorporate into the supervision process those excellent teachers and researchers who rose through the profession at a time when research was regarded as a way to increase knowledge and improve patient care, not just a gateway to an academic career or a cash-cow for universities.

1. Health Professions Council of South Africa Subcommittee for Postgraduate Education and Training (Medical). New Requirements for the Registration of Specialists in South Africa. http://www.hpcsa. org.za/downloads/medical_dental/new_requirements_for_registration_of_specialists_in_sa.pdf (accessed 24 February 2011)

2. CHE Criteria for programme accreditation, November 2004. 3.1.8 Postgraduate policies, procedures and regulations: Criterion 9 (ii). http://nr-online.che.ac.za/html_documents/CHE_accreditation_ criteria_Nov2004.pdf (accessed 28 July 2014).

3. Benatar S. Professional competence and professional misconduct in South Africa. S Afr Med I 2014;104(7):480-482. [http://dx.doi.org/10.7196/SAMJ.8492]

2014;104(7):480-482. [http://dx.doi.org/10.7196/SAM].8492]
Wingfield B. How long does it take to get a PhD? S Afr J Sci 2010;106(11/12), Art. \#469, 1 page. [http:// 4. Wingfield B. How long does it take to get
dx.doi.org/10.4102/sajs.v106i11/12.469]

dx.doi.org/10.4102/sajs.v106i11/12.469]
5. Gladwell, M. Outliers: The Story of Success. New York: Little, Brown and Company, 2008.

6. The Higher Education Qualification Sub-framework. Qualifications, volumes of learning and credits, para 29, p. 15. CHE 2013. http://www.che.ac.za/sites/default/files/publications/HEQSF\%202013.pdf (accessed 17 July 2014).

7. Wingfield B. How much time does it take to supervise a PhD student? S Afr J Sci 2012;108(11-12), Art. \#1454, 2 pages. [http://dx.doi.org/10.4102/sajs.v108i11/12.1454]

8. Prince MJ, Felder RM, Brent R. Does faculty research improve undergraduate teaching? An analysis of existing and potential synergies. Journal of Engineering Education 2007;96(4):283-294. [http://dx.doi. org/10.1002/j.2168-9830.2007.tb00939.x] 